Check for updates

Cite this: RSC Adv., 2017, 7, 53653

\title{
Computational design of enhanced photocatalytic activity of two-dimensional cadmium iodide $\uparrow$
}

\author{
Lin Tao and Le Huang (D) *
}

The recent synthesis of two-dimensional cadmium iodide $\left(\mathrm{Cdl}_{2}\right)$ opens up the questions of its properties and potential applications in optoelectronic and photovoltaic devices. Using a first-principles design approach, the electronic structure of $2 \mathrm{D} \mathrm{Cdl}{ }_{2}$ is determined. The calculated results of the band gaps and band edges demonstrated that $\mathrm{Cdl}_{2}$ is a suitable photocatalyst for water splitting. Monolayer $\mathrm{Cdl}_{2}$ should exhibit a relatively low photocatalytic activity due to its large band gap (about $3.0 \mathrm{eV}$ ). Some favourable doping can introduce extra bands to its band gap with the redox potentials of water straddled in its band gap, which can lead to improved photocatalytic performance. Multilayer $\mathrm{Cdl}_{2}$ with a narrower band gap may absorb a finite amount of visible light, making it a more suitable photocatalyst. Furthermore, multilayer $\mathrm{Cdl}_{2}$ exhibits significant modulation of its band gap and band alignment by applying normal strain and a vertical electric field. A reduced band gap with the CBM and VBM approaching the $\mathrm{H}^{+} / \mathrm{H}_{2}$ and the $\mathrm{H}_{2} \mathrm{O} / \mathrm{O}_{2}$ potentials, respectively, can result in an enhanced photocatalytic activity by applying normal strain and a vertical electric field.

Received 31st August 2017 Accepted 1st November 2017

DOI: $10.1039 / c 7 r a 09687 a$

rsc.li/rsc-advances promising alternative materials for optoelectronic applications. ${ }^{17-20}$ Furthermore, heterobilayer GeSe/SnS could have promising applications in photovoltaic devices by modifying its electronic structure. ${ }^{21}$ These $2 \mathrm{D}$ materials maximize the surface area available for water splitting and minimize the distance of generated electron and hole migration, which enhances their catalytic performance. $^{22}$

Beyond 2D chalcogenide materials, metal halide materials with layered crystal structures (e.g., $\mathrm{PbI}_{2}$ and $\mathrm{CdI}_{2}$ ) have also attracted significant attention due to their importance as precursors for the synthesis of halide perovskites. ${ }^{23-27}$ Our previous works have demonstrated that layered $\mathrm{PbI}_{2}$ possesses great potential in photovoltaic devices. ${ }^{28,29} 2 \mathrm{D} \mathrm{CdI}_{2}$ nanoplates have also been synthesized by using a vapor transport and deposition approach. ${ }^{25,30,31}$ However, the physical properties of $2 \mathrm{D} \mathrm{CdI}_{2}$ are far from clear. In particular, we focus on layered $\mathrm{CdI}_{2}$ as a possible photocatalyst and propose some effective approaches to improve its photocatalytic performance.

\section{Computational methods}

All calculations are implemented in the VASP $\operatorname{code}^{32,33}$ within the projector-augmented plane-wave method. ${ }^{34}$ The generalized gradient approximation (GGA) of the Perdew, Burke and Ernzerhof (PBE) functional ${ }^{35}$ is adopted for the electron exchange and correlation. The plane-wave cutoff energy is set as $450 \mathrm{eV}$ and the HSE06 hybrid functional ${ }^{36-38}$ is used to calculate the band structure of monolayer $\mathrm{CdI}_{2}$. A vacuum larger than $15 \AA$ is used to simulate the isolated sheet. The pair-wise force field in the DFT-D2 method of Grimme ${ }^{39}$ is employed in the
School of Materials and Energy, Guangdong University of Technology, Guangzhou, Guangdong 510006, China. E-mail: huangle@semi.ac.cn

$\dagger$ Electronic supplementary information (ESI) available. See DOI: 10.1039/c7ra09687a 
calculations of multilayer $\mathrm{CdI}_{2}$ to correctly describe the vdW interactions between layers. For the relaxation of $\mathrm{CdI}_{2}$ of various thicknesses, a $(15 \times 15 \times 1)$ Monkhorst-Pack grid $^{40}$ is used, whereas for the relaxation of a doped $\mathrm{CdI}_{2}$ monolayer, the first Brillouin zone is sampled with a $(5 \times 5 \times 1)$ Monkhorst-Pack grid. All structures are fully relaxed with a force tolerance of $0.02 \mathrm{eV} \AA^{-1}$.

\section{Results and discussion}

Compared with layered transition metal dichalcogenides, $\mathrm{CdI}_{2}$ has a layered structure with a hexagonal unit cell and it crystallizes in the space group $P 6_{3} m c$. This indicates that the $1 \mathrm{~T}$ phase of $\mathrm{CdI}_{2}$ is the most stable, rather than the $2 \mathrm{H}$ phase. As shown in Fig. 1a, each sheet of Cd atoms is sandwiched between two sheets of I atoms through strong ionic bonding, ${ }^{\mathbf{4 1 , 4 2}}$ and the atomic layers combined together via weak van der Waals (vdW) interactions. The optimized lattice constant of monolayer $\mathrm{CdI}_{2}$ is $4.33 \AA$. Its band structure calculated on the PBE level indicates an indirect band gap of $2.53 \mathrm{eV}$ in Fig. 1b. According to the calculated density of states (Fig. 1c), the VBM is mainly contributed to by Cd_p and I_p orbitals and the CBM is composed of I_p and Cd_s orbitals. In order to correct the PBE band gaps, a hybrid Heyd-Scuseria-Ernzerhof functional is adopted to describe the electron exchange and correlation (Fig. S1†). The general features of the band structure are well preserved except for a larger band gap than that of the PBE functional. Furthermore, the spin-orbit coupling (SOC) effect is taken into consideration in Fig. S1. $\dagger$ An obvious spin-orbit splitting is observed at the VBM of the band structure. As a result, the initial quadruple degenerate VBM becomes doubly degenerate. Meanwhile, the SOC exerts little influence on the
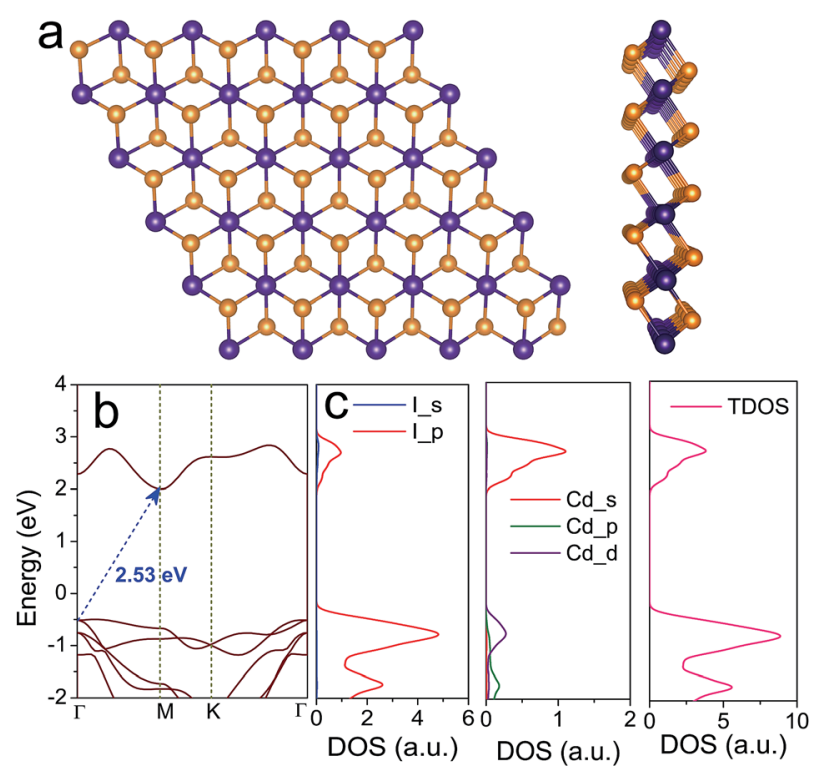

Fig. 1 (a) The lattice structure of single-layer $\mathrm{Cdl}_{2}$ from top and side views. The purple and brown balls denote the $\mathrm{Cd}$ and I atoms, respectively. (b) The band structure of single-layer $\mathrm{Cdl}_{2}$ and (c) the projected density of states (PDOS) and total density of states (TDOS).
CBM of the $\mathrm{CdI}_{2}$ bands. This is attributed to the strong coupling between the Cd_d and I_p orbitals at the VBM and the absence of the Cd_d orbital at the CBM in the band structure of $\mathrm{CdI}_{2}$. Although an indirect band gap is not favourable for water splitting, layered $\mathrm{CdI}_{2}$ has suitable band edges to straddle the redox potentials of water. The photocatalytic activity can be enhanced when combining $\mathrm{CdI}_{2}$ with other photocatalysts, such as $\mathrm{C}_{3} \mathrm{~N}_{4}$, to construct a direct $Z$-scheme type photocatalysis system for water splitting. ${ }^{\mathbf{4 3 , 4 4}}$ What is more, the imaginary part of the dielectric function and light absorption (Fig. S2†) indicate good absorption in solar light. Our results are in good agreement with previous works except for an energy shift that is due to the underestimation of the GGA-PBE functional. $\mathrm{CdI}_{2}$ thin films are reported by experimental works to have a direct optical energy gap of $\sim 3.5 \mathrm{eV}$ which seems too large for photocatalysis. $^{30,31}$ It is indicated by our results and previous experimental works that $\mathrm{CdI}_{2}$ exhibits good absorption in ultraviolet light and has great potential in photocatalysis. Efficient ways to modulate its band edges are desired to improve the optoelectronic properties and the photocatalytic activity.

It seems that the band gap of monolayer $\mathrm{CdI}_{2}$ is too large to efficiently utilize the visible light in photocatalysis. It is well known that the electronic properties including the band gaps of most layered transition metal dichalcogenides are strongly dependent on the thickness of the materials. ${ }^{45,46}$ Thus we checked the thickness-dependent electronic structure of $\mathrm{CdI}_{2}$ as shown in Fig. 2a and b. It is indicated that both the CBM and the VBM approach the Fermi level, resulting in a decrease of the band gap of $\mathrm{CdI}_{2}$ with thickness. The vacuum level is taken as a reference in Fig. 2b. It is indicated in Fig. $2 \mathrm{~b}$ that the energies of both the CBM and the VBM increase with the number of layers. Furthermore, the band gap decreases gradually with the number of layers. The evolution of the band edges and band gap with the thickness is not very significant because of the rather weak interlayer coupling. What is more, the redox potentials of water are straddled between the band edges of $\mathrm{CdI}_{2}$ with various thicknesses. The reduced band gap can make $\mathrm{CdI}_{2}$ a good photocatalyst with it absorbing solar light over a wider wavelength range. Consequently, the photocatalytic performance can be improved to some extent by increasing the thickness of $\mathrm{CdI}_{2}$ to a suitable value.

According to Fig. 2a and b, the band edges and band gap of $\mathrm{CdI}_{2}$ exhibit weak dependence on the thickness with respect to most 2D transition metal dichalcogenides. An efficient approach to improve its photocatalytic performance is desired. It has been demonstrated that $2 \mathrm{D}$ materials exhibit significant modulation of their electronic structures by applying a normal strain. The evolution of the band structures of bilayer $\mathrm{Cd}_{2}$ with an applied normal strain is shown in Fig. S3. $\uparrow$ The normal strain is calculated as $\varepsilon=\left(d_{0}-d\right) / d_{0}$. Our results indicate that the band gap of bilayer $\mathrm{CdI}_{2}$ decreases with the applied normal strain (Fig. 2c). Clear insight can be obtained from the variation of the band edges of bilayer $\mathrm{CdI}_{2}$ with the applied normal strain (Fig. 2d). The VBM increases gradually with the applied strain while the CBM does not show a monotonous trend because of the CBM's shift from the $M$ point to the $\Gamma$ point. Furthermore, the redox potentials of water are always straddled between the 

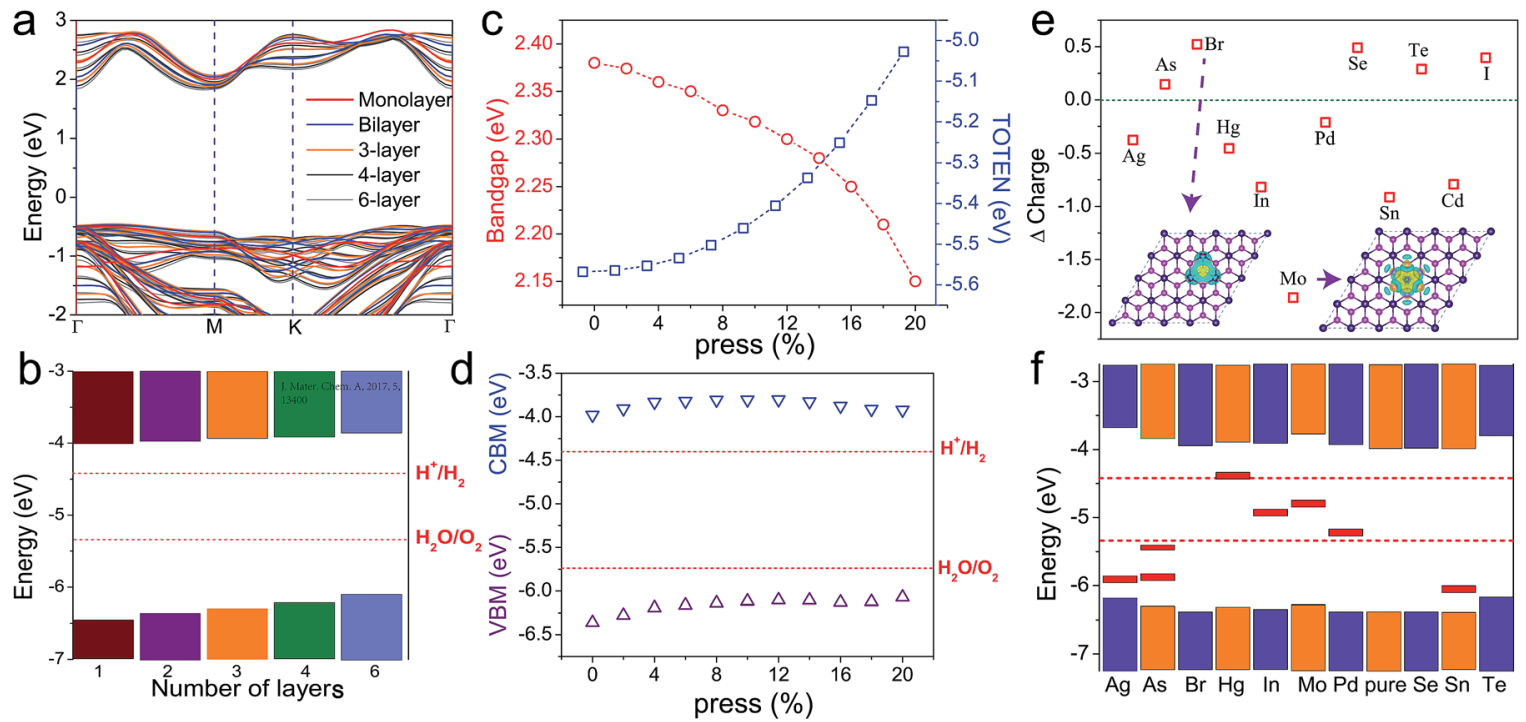

Fig. 2 (a) Band structures and (b) band alignments of $\mathrm{Cdl}_{2}$ of different thickness. Evolution of (c) band gap (red circles), total energy (blue squares) and (d) band edges of the $\mathrm{Cdl}_{2}$ bilayer as functions of the applied normal strain. (e) Transfer charge results from Bader charge analysis in the doped $\mathrm{Cdl}_{2}$ monolayer. The insets are the charge density difference in the Mo-doped and the Br-doped $\mathrm{Cdl}_{2}$ monolayer. (f) Band alignments of the doped $\mathrm{Cdl}_{2}$ monolayer. The red stripes in the band gap are the dopant bands introduced by substitution. The vacuum level is set as zero and is taken as a reference in (b), (d), and (f). The redox potentials of water are plotted as red dashed lines.

VBM and the CBM of the pressed $\mathrm{CdI}_{2}$ bilayer. The $\mathrm{CdI}_{2}$ bilayer with a reduced band gap from the application of normal strain is a good photocatalyst with improved performance due to the widened wavelength range for light absorption.

It should be mentioned that the interlayer binding in the $\mathrm{CdI}_{2}$ bilayer is relatively weaker than that in most of the transition metal dichalcogenide bilayers such as those of $\mathrm{MoS}_{2}$. Fig. 2c also shows the evolution of the total energy of the $\mathrm{CdI}_{2}$ bilayer. The charge density difference (Fig. S4†) shows slight charge transfer between layers in the $\mathrm{CdI}_{2}$ bilayer upon binding together. The weak binding gives rise to the relatively weak response of the electronic structure and strain energy to the applied strain. Similar evidence comes from the integrated charge density of the $\mathrm{CdI}_{2}$ bilayer under different normal strain (Fig. S5 $\dagger$ ). It is found that applying a normal strain will bring about extra charge exchanging between layers. It can be concluded that applying a normal strain is an efficient way to improve the photocatalytic performance.

Besides applying normal strain, substituted doping has also been demonstrated to be an effective method to modulate the band alignment of a semiconductor. In Fig. 2e and f, several non-magnetic atoms are employed to make substituting dopants in a $(4 \times 4 \times 1) \mathrm{CdI}_{2}$ monolayer supercell. Dopant bands are induced either in the band gap or near the band edges (Fig. S6†), which should exert a significant influence on the optoelectronic and photovoltaic properties of monolayer $\mathrm{CdI}_{2}$. In Fig. 2e, Bader charge analysis demonstrates that charge transfer occurs between dopants and it defected the $\mathrm{CdI}_{2}$ monolayer.

The band alignments of these doped $\mathrm{CdI}_{2}$ monolayers are plotted in Fig. 2f. It is found that In, Mo and Pd will introduce dopant bands in the redox potential of water, making the corresponding doped $\mathrm{CdI}_{2}$ not suitable for photocatalysis, while other dopants employed here will not introduce extra bands in the redox potential of water. $\mathrm{Ag}$, As and $\mathrm{Sn}$ dopants will induce extra bands between the VBM and the $\mathrm{H}_{2} \mathrm{O} / \mathrm{O}_{2}$ potential, and $\mathrm{Hg}$ can induce extra bands between the $\mathrm{H}^{+} / \mathrm{H}_{2}$ potential and the CBM, making the corresponding doped $\mathrm{CdI}_{2}$ suitable for photocatalysis in water splitting.

In many optoelectronic and photovoltaic devices, the materials are always subjected to a vertical electric field $\left(E_{\perp}\right)$. Multilayer 2D materials usually exhibit significant modulation of their electronic structures by applying an $E_{\perp}$. As shown in Fig. 3a, the band structures of the $\mathrm{CdI}_{2}$ bilayer under different $E_{\perp}$ are calculated. Our results indicate that its band gap is significantly reduced by the applied $E_{\perp}$. Further insight can be obtained from the variation of the band edges with the $E_{\perp}$ in Fig. 3 b. It is clear that the CBM exhibits a linear descending trend with the $E_{\perp}$ while the VBM is hardly influenced by the $E_{\perp}$. As a photocatalyst, when subjected to a vertical electric field, the $\mathrm{CBM}$ of $\mathrm{CdI}_{2}$ will approach the $\mathrm{H}^{+} / \mathrm{H}_{2}$ level. This will improve the absorption of solar light, further leading to enhanced photocatalytic activity. However, a strong $E_{\perp}$ will reduce the CBM of $\mathrm{CdI}_{2}$ to the position below the $\mathrm{H}^{+} / \mathrm{H}_{2}$ level, making $\mathrm{CdI}_{2}$ unsuitable as a photocatalyst.

To make the distinct responses of the VBM and the CBM to the applied $E_{\perp}$ clear, the band decomposed charge density of the CBM and the VBM at the four high-symmetry $k$-points $(T, M$, $K, A)$ is shown in Fig. $3 \mathrm{c}$ and d. $A$ is the $k$-point between $K$ and $\Gamma$, which is given in Fig. 3a. As can be seen, the lowest-energy carriers (holes) are almost completely localized around the I atoms, forming $\pi$ bonds. The absence of a polarized distribution between the I and Cd atoms results in the weak response of the VBM to the applied $E_{\perp}$, while at the CBM, the lowest-energy 

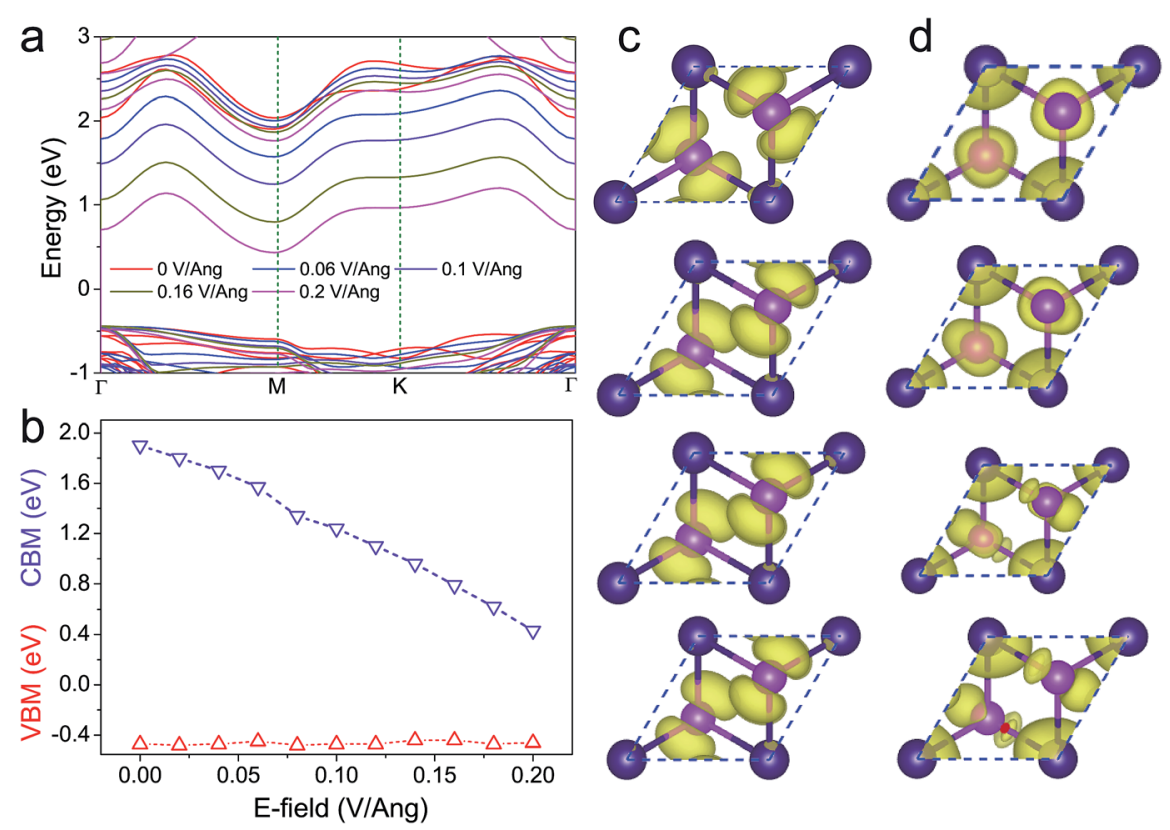

Fig. 3 Evolution of the (a) band structure and (b) band edges of the $\mathrm{Cdl}_{2}$ bilayer as functions of the applied vertical electric field. (c) and (d) plot

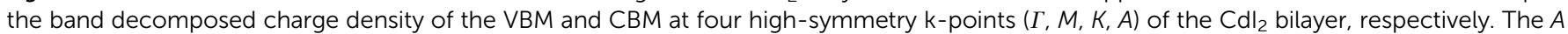
point is the $k$-point between the $K$ and the $T$ points.

carriers (electrons) are neither localized on the I atoms nor the $\mathrm{Cd}$ atoms. Charge exchange exists between I and Cd atoms. As a result, the applied $E_{\perp}$ exerts a significant influence on the CBM.

\section{Conclusions}

In summary, the electronic structure of $2 \mathrm{D} \mathrm{CdI}_{2}$ is explored by density functional theory calculations. The calculated results of the band gaps and band edges demonstrated that $\mathrm{CdI}_{2}$ is a suitable photocatalyst for water splitting. Monolayer $\mathrm{CdI}_{2}$ should exhibit a low photocatalytic activity due to its large band gap (about $3.0 \mathrm{eV}$ ). Multilayer $\mathrm{CdI}_{2}$ with a narrower band gap may absorb a finite amount of visible light, making it a more suitable photocatalyst. What is more, its photocatalytic activity can be enhanced by applying normal strain and a vertical electric field with two distinct mechanisms. Both the CBM and VBM of bilayer $\mathrm{CdI}_{2}$ can be tuned by applying a normal strain, while a vertical $E_{\perp}$ is only able to modulate the CBM of bilayer $\mathrm{CdI}_{2}$. Some favourable substituting dopants can also make the band alignment of $\mathrm{CdI}_{2}$ more suitable for photocatalysis. These results provide valuable guidance for enhancing the photocatalytic activity of $2 \mathrm{D} \mathrm{CdI} 2$.

\section{Conflicts of interest}

There are no conflicts to declare.

\section{Acknowledgements}

This work was financially supported by the National Natural Science Foundation of China (Grant no. 11674310, 61622406,
61571415, 51502283, and 51602065), the National Key Research and Development Program of China (Grant No. 2016YFB0700700), "Hundred Talents Program" of Chinese Academy of Sciences (CAS), and the CAS/SAFEA International Partnership Program for Creative Research Teams.

\section{References}

1 T. Simon, N. Bouchonville, M. J. Berr, A. Vaneski, A. Adrovic, D. Volbers, R. Wyrwich, M. Döblinger, A. S. Susha, A. L. Rogach, et al., Nat. Mater., 2014, 13, 1013.

2 X. Chen, L. Liu, Y. Y. Peter and S. S. Mao, Science, 2011, 331, 746.

3 R. Asahi, T. Morikawa, H. Irie and T. Ohwaki, Chem. Rev., 2014, 114, 9824.

4 J. Ran, J. Zhang, J. Yu, M. Jaroniec and S. Z. Qiao, Chem. Soc. Rev., 2014, 43, 7787.

5 Y. Gai, J. Li, S.-S. Li, J.-B. Xia and S.-H. Wei, Phys. Rev. Lett., 2009, 102, 036402.

6 J. Kang, S. Tongay, J. Zhou, J. Li and J. Wu, Appl. Phys. Lett., 2013, 102, 012111.

7 V. Chakrapani, J. C. Angus, A. B. Anderson, S. D. Wolter, B. R. Stoner and G. U. Sumanasekera, Science, 2007, 318, 1424.

8 A. K. Singh, K. Mathew, H. L. Zhuang and R. G. Hennig, J. Phys. Chem. Lett., 2015, 6, 1087.

9 S. Chen and L.-W. Wang, Chem. Mater., 2012, 24, 3659.

10 W. J. Ong, Front. Mater., 2017, 4, 11.

11 D. Zeng, L. Xiao, W. J. Ong, P. Wu, H. Zheng, Y. Chen and D. L. Peng, J. Mater. Chem. A, 2017, 5, 16171-16178.

12 X. Lv, W. Wei, Q. Sun, F. Li, B. Huang and Y. Dai, Appl. Catal., $B, 2017,217,275$. 
13 H. L. Zhuang and R. G. Hennig, Chem. Mater., 2013, 25, 3232. 14 H. L. Zhuang and R. G. Hennig, J. Phys. Chem. C, 2013, 117, 20440.

15 N. Singh, G. Jabbour and U. Schwingenschlögl, Eur. Phys. J. $B, 2012,85,392$.

16 G. Guo and W. Liang, J. Phys. C: Solid State Phys., 1986, 19, 995.

17 P. D. Antunez, J. J. Buckley and R. L. Brutchey, Nanoscale, 2011, 3, 2399.

18 A. K. Singh and R. G. Hennig, Appl. Phys. Lett., 2014, 105, 042103.

19 Z. Ma, B. Wang, L. Ou, Y. Zhang, X. Zhang and Z. Zhou, Nanotechnology, 2016, 27, 415203.

20 L. Huang, F. Wu and J. Li, J. Chem. Phys., 2016, 144, 114708.

21 C. Xia, J. Du, W. Xiong, Y. Jia, Z. Wei and J. Li, J. Mater. Chem. A, 2017, 5, 13400.

22 A. Kudo and Y. Miseki, Chem. Soc. Rev., 2009, 38, 253.

23 Y. Hu, Y. Guo, Y. Wang, Z. Chen, X. Sun, J. Feng, T. M. Lu, E. Wertz and J. Shi, J. Mater. Res., 2017, 325, 1.

24 J. Zhang, T. Song, Z. Zhang, K. Ding, F. Huang and B. Sun, J. Mater. Chem. C, 2015, 3, 4402.

25 R. Ai, X. Guan, J. Li, K. Yao, P. Chen, Z. Zhang, X. Duan and X. Duan, ACS Nano, 2017, 11, 3413.

26 I. Karbovnyk, I. Bolesta, I. Rovetskyi, V. Lesivtsiv, Y. Shmygelsky, S. Velgosh and A. Popov, Low Temp. Phys., 2016, 42, 594.

27 J. Robertson, J. Phys. C: Solid State Phys., 1979, 12, 4753.

28 M. Zhong, S. Zhang, L. Huang, J. You, Z. Wei, X. Liu and J. Li, Nanoscale, 2017, 9, 3736.
29 M. Zhong, L. Huang, H.-X. Deng, X. Wang, B. Li, Z. Wei and J. Li, J. Mater. Chem. C, 2016, 4, 6492.

30 P. Tyagi, A. G. Vedeshwar and N. C. Mehra, Phys. B, 2001, 304, 166.

31 I. A. Kariper, J. Mater. Res. Technol., 2016, 5, 77.

32 G. Kresse and J. Furthmüller, Phys. Rev. B, 1996, 54, 11169.

33 G. Kresse and J. Furthmüller, Comput. Mater. Sci., 1996, 6, 15.

34 P. E. Blöchl, Phys. Rev. B, 1994, 50, 17953.

35 J. P. Perdew, K. Burke and M. Ernzerhof, Phys. Rev. Lett., 1996, 77, 3865.

36 J. Heyd, G. E. Scuseria and M. Ernzerhof, J. Chem. Phys., 2003, 118, 8207.

37 J. Heyd, J. E. Peralta, G. E. Scuseria and R. L. Martin, J. Chem. Phys., 2005, 123, 174101.

38 J. Heyd, G. E. Scuseria and M. Ernzerhof, J. Chem. Phys., 2006, 124, 9906.

39 S. Grimme, J. Comput. Chem., 2006, 27, 1787.

40 H. J. Monkhorst and J. D. Pack, Phys. Rev. B, 1976, 13, 5188.

41 I. A. Kariper, J. Mater. Res. Technol., 2016, 5, 77.

42 Y. Wang, Y.-Y. Sun, S. Zhang, T.-M. Lu and J. Shi, Appl. Phys. Lett., 2016, 108, 013105.

43 J. Wang, Z. Guan, J. Huang, Q. Li and J. Yang, J. Mater. Chem. A, 2014, 2, 7960.

44 Q. Li, N. Zhang, Y. Yang, G. Wang and D. H. Ng, Langmuir, 2014, 30, 8965.

45 W. Jin, P.-C. Yeh, N. Zaki, D. Zhang, J. T. Sadowski, A. AlMahboob, A. M. van Der Zande, D. A. Chenet, J. I. Dadap, I. P. Herman, et al., Phys. Rev. Lett., 2013, 111, 106801.

46 R. Coehoorn, C. Haas, J. Dijkstra, C. Flipse, R. De Groot and A. Wold, Phys. Rev. B, 1987, 35, 6195. 